\title{
Pseudomorphic modulation-doped AlGaAs/InGaAs/GaAs heterostructures with strong manifestation of many-body effects
}

\author{
W.T. Masselink, H. Kissel, U.Mueller, C. Walther \\ Humboldt-Universitat zu Berlin, Dept. of Physics, Invalidenstrasse 110, D-10115 Berlin, Germany
}

Yu.I. Mazur,G.G .Tarasov, G.Yu. Rud'ko, M.Ya. Valakh,V. Malyarchuk, Z.Ya. Zhuchenko

Institute of Semiconductor Physics, National Academy of Sciences, Prospect Nauki 45, 03650 Kiev, Ukraine

\begin{abstract}
Photoluminescence (PL) study of pseudomorphic heavily modulation-doped $\mathrm{Al}_{\mathrm{x}} \mathrm{Ga}_{1-\mathrm{x}} \mathrm{As} / \mathrm{In}_{\mathrm{y}} \mathrm{Ga}_{1-\mathrm{y}} \mathrm{As} / \mathrm{GaAs}$ heterostructures shows fundamental changes in the PL spectrum under excitation pumping and/or temperature increase. In most the high and low energy tails of the PL feature undergo the principal transformations. High-energy tail peculiarities are related to the repelling of the Fermi-edge singularity (FES) and the excitonic states. The character of repelling depends crucially on the excitation density and/or temperature. At low temperature the origination of the FES feature has been observed for the first time under increasing the excitation density. The FES appearance is accompanied by the formation of an abrupt high energy edge and occurs far below by intensity the hybridized $n=2$ exciton manifestation. Strong screening of the $n=2$ exciton state by photoexcited carriers is observed, resulting in the $2 \mathrm{D}$ electron gas-heavy hole recombination for the second electron subband. The many-body feature is detected in the magnetoluminescence spectrum. This feature develops in magnetic field $B=7 \mathrm{~T}$ at low temperature $(T=4.2 \mathrm{~K})$ and is surely detected up to $T \approx 50 \mathrm{~K}$. The LO-phonon side bands for the parent transitions between the Landau levels (LL's) are revealed in the low-energy tail of the PL spectra in magnetic field. The evolution of these phonon side-band with temperature and excitation density is observed. The enhanced strength of phonon side-bands is attributed to an enhanced Frohlich coupling with account of confined phonon and interface modes.
\end{abstract}

Keywords: photoluminescence, Fermi-edge singularity, magnetic field, Frohlich coupling, $\mathrm{Al}_{\mathrm{x}} \mathrm{Ga}_{1-\mathrm{x}} \mathrm{As} / \mathrm{In}_{\mathrm{y}} \mathrm{Ga}_{1-\mathrm{y}} \mathrm{As} / \mathrm{GaAs}$ heterostructures.

Paper received 01.09.99; revised manuscript received 21.11.99; accepted for publication 14.03.00.

\section{Introduction}

Pseudomorphic modulation-doped $\mathrm{Al}_{\mathrm{x}} \mathrm{Ga}_{1-\mathrm{x}} \mathrm{As} / \mathrm{In}_{\mathrm{y}} \mathrm{Ga}_{1-}$ y As/GaAs heterostructures benefit from a higher electron mobility and saturation velocity in the InGaAs quantum well (QW) channel accompanied by better confinement properties and higher two-dimensional electron gas (2DEG) density when compared to conventional $\mathrm{Al}_{\mathrm{x}} \mathrm{Ga}_{1-\mathrm{x}} \mathrm{As} / \mathrm{GaAs}$ heterostructures [1-3]. They provide nearly ideal conditions for the study of Landau quantization, valence-band complexities, screening, many-body effects, such as, band-gap renormalization, Fermi-edge singularity (FES), shake-up processes and so on [4-8]. In order to observe the FES and the excitonic enhancement associated with the second subband in asymmetric modulation-doped QW's the electron gas density has to be adjusted in an appropriate way: the Fermi level should be placed in close proximity of the $n=2$ electron level. Besides, the strong overlap of the wave functions for the spatially separated electron and hole is necessary to ensure the effective electron-hole correlation.

Typically the FES is identified:

i) in photoluminescence (PL) as an enhancement of oscillator strength for transitions close to the Fermi edge when the disorder or hole localization violate the momentum conservation law and allow the transitions nominally forbidden by this law;

ii) in absorption it appears as an excitonic enhancement by a factor of 2 at $4 \mathrm{~K}$ for electron density of the order of $(1 \ldots 5) \cdot 10^{11} \mathrm{~cm}^{-2}$ with subsequent decreasing at higher densities [9].

All aforementioned effects demonstrate strong temperature, excitation density and magnetic field dependences. So, Chen et al. [10] have observed in perpendicular 


\section{W.T. Masselink et al.: Pseudomorphic modulation-doped AlGaAs/InGaAs/GaAs heterostructures...}

magnetic field $B$ very strong periodic in $B^{-1}$ intensity oscillations for PL at low temperature caused by the excitonic resonant enhancement of the FES in the highest occupied Landau level (LL). The evidence for a FES has been obtained from the temperature dependence of the $n=2$ related peak of $\mathrm{PL}$ in $\mathrm{Al}_{\mathrm{x}} \mathrm{Ga}_{1-\mathrm{x}} \mathrm{As} / \mathrm{In}_{\mathrm{y}} \mathrm{Ga}_{1-\mathrm{y}} \mathrm{As} /$ GaAs [3]. This finding was latter argued because the pronounced temperature dependence of the $n=2$ related peak, caused by the intersubband transitions occurring close to the Fermi energy $E_{F}$ in $\mathrm{Al}_{\mathrm{x}} \mathrm{Ga}_{1-\mathrm{x}} \mathrm{As} / \mathrm{In}_{\mathrm{y}} \mathrm{Ga}_{1-\mathrm{y}} \mathrm{As} /$ GaAs (when $E_{F} \cong E_{2}-E_{1}$ ), can be ascribed also to changes in the electron and exciton distributions alone [9]. It was concluded [9] that only in the case when the both features, $E_{F}$ and $n=2$ related, are present in the PL spectrum the many-body nature of the peak at $E_{F}$ can be firmly stated. The behavior of the FES and the $n=2$ exciton under the near-resonance condition between the $n=2$ electronic subband and the $E_{F}$ has been recently studied in $\mathrm{Al}_{\mathrm{x}} \mathrm{Ga}_{1-}$ ${ }_{x} \mathrm{As} / \mathrm{In}_{\mathrm{y}} \mathrm{Ga}_{1-\mathrm{y}} \mathrm{As} / \mathrm{GaAs}$ by means of PL and PL excitation (PLE) with temperature and/or excitation density variation [11]. It was demonstrated unequivocally that the FES quenches rapidly with temperature increasing whereas the magnitude of the $n=2$ exciton feature grows progressively accompanied by a blueshift of the energy position.

However, the real physical picture for the FES observation seems to be even more complicated. It has been found recently that the FES appearance is critically dependent on the 2DEG concentration [12]. The FES observation is prohibited at definite, comparatively low electron concentration, reflecting inherent properties of the QW structures, influenced by many-body interactions. The many-body excitonic transition is actually contributed by the scattering of electrons by holes and the strong renormalization of the electron self-energy [13,14]. The exciton enhancement is shown to survive in the low-temperature region $(T \leq 50 \mathrm{~K})$ [15]. Nevertheless, the Fermi edge transitions were observed even at $T=152 \mathrm{~K}$ (for reference see [16]). Moreover, the calculations [16] using the self-consistent Hartree approximation with account of the many-body effects, phonon and impurity scattering, strain effects, and the interface imperfection, have shown that even for temperatures as high as $T=351 \mathrm{~K}$ the many-body corrections in absorption spectra are still observable.

We observed in our previous studies [8,17] of pseudomorphic modulation-doped $\mathrm{Al}_{\mathrm{x}} \mathrm{Ga}_{1-\mathrm{x}} \mathrm{As} / \mathrm{In}_{\mathrm{y}} \mathrm{Ga}_{1-\mathrm{y}} \mathrm{As} / \mathrm{GaAs}$ heterostructures $(x=0.2, y=0.1)$ a characteristically different PL behavior, depending on the InGaAs QW width. There we restrained ourselves with the case of the first electron subband filling, a low 2DEG density, and low temperatures. It has been developed the technique which allows us to detect the localized hole states through the optical detection of quantum oscillations of PL in magnetic field. At least, two sorts of localized holes available in our heterostructures, favor the observation of many-body effects. In order to meet the favorable conditions of the FES observation now the electron density is increased to place the Fermi level nearby the $n=2$ electronic level, and the PL measurements with and without magnetic field are performed in a wide temperature region $(4.2-120 \mathrm{~K})$ and at various excitation densities. We present here new data on the PL behavior in the spectral range of $E_{F}$ and $E_{n=2}$, which can be interpreted in terms of the FES origination. Magnetoluminescence data give evidence to the phonon side-bands of LL in heavily doped heterostructures. The optical characterization of the QW structures under investigation is performed, using both the low and high temperature PL data.

\section{Experimental details}

The samples used in this investigation are pseudomorphic $\mathrm{In}_{\mathrm{y}} \mathrm{Ga}_{1-\mathrm{y}} \mathrm{As}$ modulation-doped QW's grown on semi-insulating, (100)-oriented GaAs substrates in a Riber 32-P gas-source MBE (GSMBE) system. The typical epitaxial layer sequence consists of a GaAs buffer layer, an undoped $\mathrm{In}_{\mathrm{y}} \mathrm{Ga}_{1-\mathrm{y}} \mathrm{As}$ strained QW (2DEG channel), an $\mathrm{Al}_{\mathrm{x}} \mathrm{Ga}_{1-\mathrm{x}} \mathrm{As}$ undoped spacer, an $\mathrm{Al}_{\mathrm{x}} \mathrm{Ga}_{1-\mathrm{x}} \mathrm{As}$ heavily Sidoped supplier layer, and a Si-doped GaAs cap layer. The $\mathrm{Al}_{\mathrm{x}} \mathrm{Ga}_{1-\mathrm{x}} \mathrm{As}$ and $\mathrm{In}_{\mathrm{y}} \mathrm{Ga}_{1-\mathrm{y}} \mathrm{As}$ layer compositions were chosen to be: $x=0.20$ and $y=0.19$, respectively. The QW thickness is of $15 \mathrm{~nm}$. Double-crystal X-ray diffraction and simulation of the double X-ray rocking curve were used to verify the samples' structural parameters. The measurements were carried out using a Bede Q1B Xray diffractometer using the symmetric (004) reflex. The $\mathrm{X}$-ray patterns were indicative of the high quality of the structures under investigation. The electron sheet density $N_{s}$ of the 2D electrons is obtained from low-field Hall measurements down to $4.2 \mathrm{~K}\left(N_{s}=11.8 \cdot 10^{11} \mathrm{~cm}^{-2}\right)$. The PL was excited by the $514.5 \mathrm{~nm}$ line of a cw $\mathrm{Ar}^{+}$laser. The PL signal was dispersed through a 3/4-meter CzernyTurner scanning spectrometer, with a spectral resolution better than $0.1 \mathrm{meV}$, and detected using phase-sensitive detection with a thermoelectrically cooled photomultiplier tube [containing a $\mathrm{GaAs}(\mathrm{Cs})$ photocathode] or a $\mathrm{LN}_{2}$ cooled high-purity Ge detector. The samples were mounted in an Oxford Spectromag 4000 system, which allows measurements in magnetic fields up to $7 \mathrm{~T}$ and at temperatures from 1.7 to $300 \mathrm{~K}$.

\section{Results and discussions}

\section{A. PL in zero magnetic field}

In the following we present the results of PL study for pseudomorphic modulation-doped $\mathrm{Al}_{\mathrm{x}} \mathrm{Ga}_{1-\mathrm{x}} \mathrm{As} / \mathrm{In}_{\mathrm{y}} \mathrm{Ga}_{1-}$ ${ }_{\mathrm{y}} \mathrm{As} / \mathrm{GaAs}$ heterostructures, where two spectral features become developed: a strong peak originated from the recombination of photoexcited holes, thermalized in the $n$ $=1$ heavy-hole valence subband, and electrons in the $n=$ 1 conduction subband ( $E_{11}$-transition); a weak feature caused by arising from the transitions from the $n=2$ conduction subband to $n=1$ heavy-hole subband, which is observed either at elevated temperatures and/or under an increased laser excitation ( $E_{21}$-transition). As far as the Fermi energy is close to the $E_{2}$ energy level (but be- 
W.T. Masselink et al.: Pseudomorphic modulation-doped AIGaAs/InGaAs/GaAs heterostructures...

low it), the hybridization mechanisms are expected to be actual, resulting in an enhancement at $E_{F}$. Typically if the $E_{F}$ (measured from the bottom of the first conduction subband) is placed far below the $E_{2}$ the emission spectrum represents a single peak, asymmetrically broadened towards the high-energy side. The pronounced FES develops in the presence of strong disorder within QW, resulting in the strong hole localization. Otherwise the FES singularity is basically weak, being generated from the infinitely many low-frequency electron-hole pair excitations of the Fermi sea. When the $E_{F}$ falls in the close vicinity of the $E_{2}$-level, the bound $n=2$ excitonic state (bare exciton) immerses into the continuum of the scattered states of the $n=1$ subband and transforms into a hybridized excitonic state of the Fano resonance sort due to the interference between the FES and the exciton [13]. The FES becomes enhanced, and the measure of enhancement is strongly dependent on the details of the intersubband interaction, in particular, on the $n=2$ electron subband and $n=1$ hole subband mixing caused by the hole potential.

In order to be more precise in further estimates, we calculate the subband structure for $\operatorname{In}_{\mathrm{y}} \mathrm{Ga}_{1-\mathrm{y}} \mathrm{As}$ quantum well in our heterostructures. The finite-difference technique is used to solve self-consistently the coupled Schrödinger and Poisson equations within the effective mass approximation with a base of cubic $\beta$ splines. The composition-dependent band gap for an unstrained $\mathrm{Al}_{\mathrm{x}} \mathrm{Ga}_{1-\mathrm{x}} \mathrm{As}$ layer is calculated using the equation $E_{g}(x)=1.5192+1.360 x+0.220 x^{2} \mathrm{eV}$ at $4 \mathrm{~K}$ [18] and $E_{g}(y)=1.5192-1.5837 y+0.470 y^{2} \mathrm{eV}$ for an unstrained $\mathrm{In}_{\mathrm{y}} \mathrm{Ga}_{1-\mathrm{y}} \mathrm{As}$ layer [19]. The band offsets which play an important role in the subband separation are not determined reliably for our heterostructures. The alignment is significantly complicated by the strain. Therefore the offsets are chosen to be composition-dependent following Joyce et al. [20], and all other material parameters are interpolated between the binary values. The Hartree potential is calculated from the Poisson equation with standard boundary conditions and the exchange-correlation potential, derived from the density-functional theory within the local-density approximation is taken in the form paramet-rized by Hedin and Lundqvist [21]. The electron sheet density is given by:

$n_{S}(z)=\frac{m^{*} k T}{\pi \hbar^{2}} \sum_{i} \ln \left[1+\exp \left(\frac{E_{F}-E_{i}}{k T}\right)\right]\left|\Psi_{i}(z)\right|^{2}$

where $m^{*}$ is the in-plane electron effective mass, $k$ is Boltzmann's constant, $T$ is temperature, and $E_{i}$ are the electron envelope function and the energy bottom of the $i$-th subband. The Fermi level for a given temperature is calculated by iteration from the charge-neutrality condition.

The calculated subband energies and the energy eigenfunctions for the $\operatorname{In}_{0.19} \mathrm{Ga}_{0.81}$ As QW are shown in Fig. 1. The Si-doping introduces a strong band-bending potential which yields to a strong modification of the wave

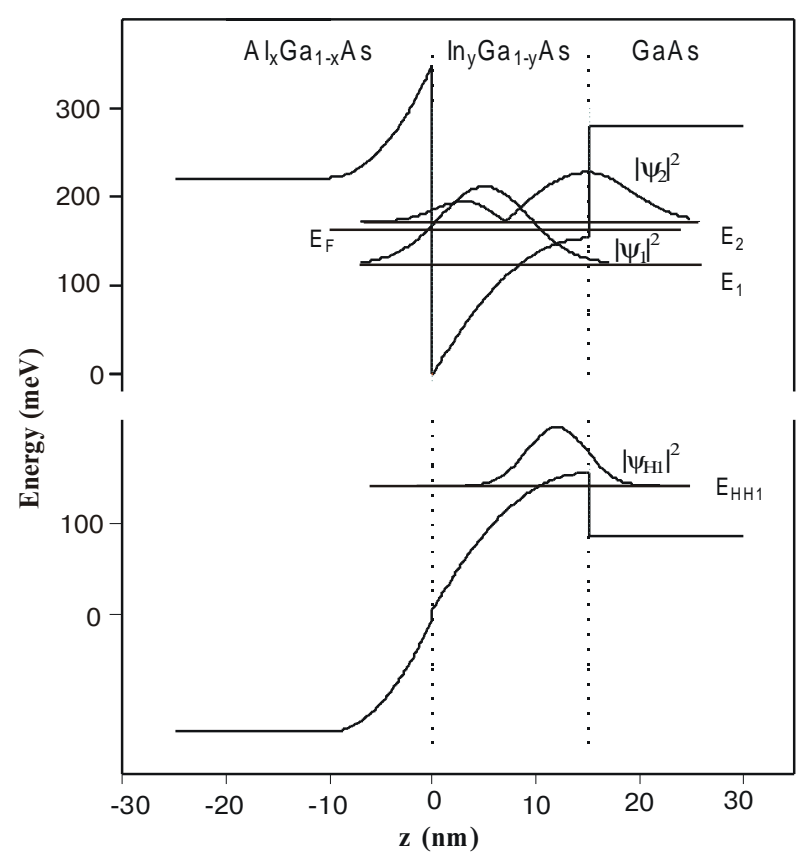

Fig. 1. Conduction and valence band profiles calculated from a coupled Schrödinger-Poisson analysis for $\mathrm{Al}_{\mathrm{x}} \mathrm{Ga}_{1-\mathrm{x}} \mathrm{As} / \mathrm{In}_{\mathrm{y}} \mathrm{Ga}_{1-\mathrm{y}} \mathrm{As} /$ GaAs heterostructure. Squared envelope functions for electrons and holes are also shown. The distance $z$ is measured from $\mathrm{Al}_{\mathrm{x}} \mathrm{Ga}_{1-\mathrm{x}} \mathrm{As} / \mathrm{In}_{\mathrm{y}} \mathrm{Ga}_{1-\mathrm{y}} \mathrm{As}$ interface position.

functions and the corresponding energy levels in the InGaAs QW as compared to an undoped QW. The $n=1$ conduction subband is filled up to the $E_{F}$. The intersubband separation $\Delta=E_{2}-E_{1}$ is calculated to be $48 \mathrm{meV}$, and $E_{F}$ is of $5 \mathrm{meV}$ smaller than the $E_{2}$ energy. One can see that the wave function of the $n=1$ electron state peaks nearby the $\mathrm{Al}_{\mathrm{x}} \mathrm{Ga}_{1-\mathrm{x}} \mathrm{As} / \mathrm{In}_{\mathrm{y}} \mathrm{Ga}_{1-\mathrm{y}} \mathrm{As}$ interface, while the wave function of the $n=2$ electron state has a node and its main peak is in proximity of the $\mathrm{In}_{\mathrm{y}} \mathrm{Ga}_{1-\mathrm{y}} \mathrm{As} /$ GaAs interface. The $n=1$ wave function of the heavy hole subband (the in-plane light hole subband $(3 / 2, \pm 3 / 2)$ is splitted off by about $60 \mathrm{meV}$ below from the in-plane heavy hole one $(3 / 2, \pm 1 / 2)$ due to the biaxially strained $\mathrm{In}_{\mathrm{y}} \mathrm{Ga}_{1-\mathrm{y}} \mathrm{As}$ ) is also positioned close to the $\mathrm{In}_{\mathrm{y}} \mathrm{Ga}_{1-\mathrm{y}} \mathrm{As} /$ GaAs interface. The wave functions calculated allow to find the ratio of the electron-hole overlap integrals squared for the $E_{21}$ and $E_{11}$ transitions. It takes the value of 12.7. Possessing the information derived from the calculations one can expect the single-peaked PL spectrum from $\mathrm{Al}_{\mathrm{x}} \mathrm{Ga}_{1-\mathrm{x}} \mathrm{As} / \mathrm{In}_{\mathrm{y}} \mathrm{Ga}_{1-\mathrm{y}} \mathrm{As} / \mathrm{GaAs}$ heterostructures under investigation.

Indeed, Fig. 2 shows a characteristic PL spectrum at $4.2 \mathrm{~K}$. The strong feature at $1.284 \mathrm{eV}$ (Fig. 2) stems from the recombination of the $n=1$ electrons as well as the $n=$ 1 heavy holes and has a typical asymmetric line shape. The recombination peaks near the $n=1$ subband bottom $(\vec{k}=0)$ and strongly decreases in intensity towards the $E_{F}$ energy. The PL line shape originates mainly from the 2D electrons scattered by randomly distributed impurities and defects, among which there are the ionized do- 
W.T. Masselink et al.: Pseudomorphic modulation-doped AlGaAs/InGaAs/GaAs heterostructures...

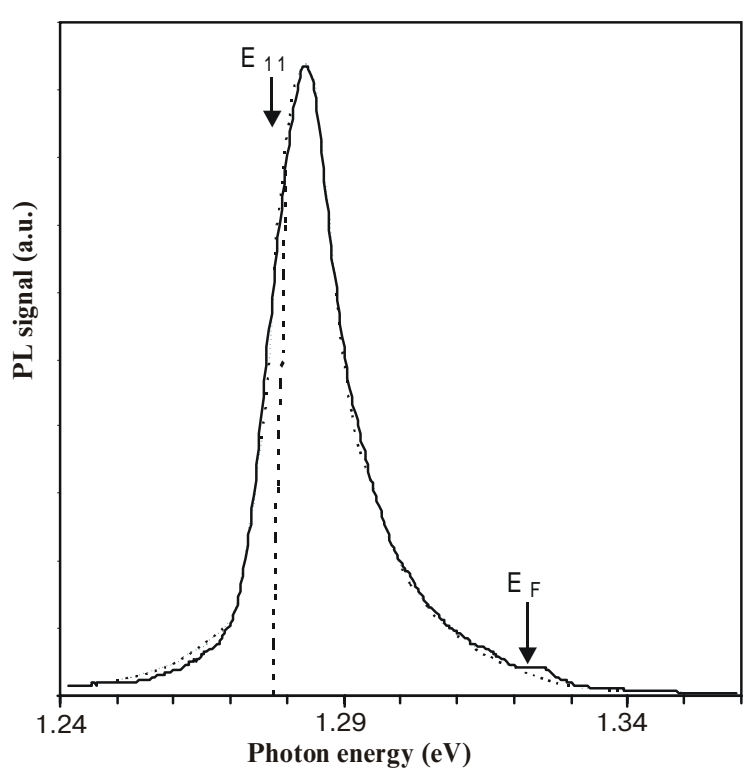

Fig. 2. PL spectrum from $\mathrm{Al}_{0.2} \mathrm{Ga}_{0.8} \mathrm{As} / \mathrm{In}_{0.19} \mathrm{Ga}_{0.81} \mathrm{As} / \mathrm{GaAs}$ heterostructure at lowest excitation power $(T=4.2 \mathrm{~K})$. Dashed line presents the fit to Eq. 2. Low-energy tail is approximated by Urbach form. Arrows stand for $E_{11}$ and $E_{F}$ energies.

nors in the $\mathrm{Al}_{\mathrm{x}} \mathrm{Ga}_{1-\mathrm{x}} \mathrm{As}$ spacer layer and the alloy fluctuations in the $\mathrm{In}_{\mathrm{y}} \mathrm{Ga}_{1-\mathrm{y}} \mathrm{As} \mathrm{QW}$. Direct allowed transitions at $(\vec{k}=0)$ are very strong in comparison with the impurity and alloy fluctuation-assisted indirect transitions. However, in the $n$-type hetero-structures only a small fraction of electrons near the bottom of the conduction subband are involved into recombination with the hole available at the top of the valence band, occupied at low temperature and low excitation density. Due to the impurity presence the momentum conservation is broken, and the transitions from electrons in states up to $k=k_{F} \sim 2 \cdot 10^{6} \mathrm{~cm}^{-1}$ become allowed. The net result is that indirect transitions for electrons with finite wave vector up to $k_{F}$ exceed by intensity the direct transitions.

The shape of spectral feature is well described in terms of the microscopic theory, developed by Lyo and Jones [22] for the steady-state PL at low temperatures in the modulation-doped degenerate direct-band-gap semiconductor QW's. Due to the theory [22] the total PL linewidth is contributed by damping effects through the collisioninduced width of carrier levels, Fermi-level effects through the impurity-assisted processes, and temperature effects through the thermal distribution of the minority carriers. The line-shape function then takes the form:

$S_{i j}(\varepsilon) \propto \int_{0}^{\varepsilon} f\left(\tau-\mu_{v j}\right) f\left(\varepsilon-\tau-\mu_{c i}\right) K(\varepsilon, \tau) d \tau$,

where $\varepsilon$ is the photon energy minus the effective band gap, $f$ is the Fermi distribution function, $\mu_{v j}$ and $\mu_{c i}$ stand for chemical potentials in $j$ th valence and $i$ th conduction bands, respectively. The Lorentzian $K$ accounts for the processes associated with direct transitions in $k$ space and indirect impurity-assisted processes. Following the procedure described in Ref. [22] the fit was performed for the line-shape of PL excited by a low-intensity cw laser if the structure at Fermi edge is not yet developed (see dashed line on Fig. 2). The set of parameters was derived from the fit: $E_{g}=1.278 \mathrm{meV}, \mathrm{G}=13.8 \mathrm{meV}$ is the full width at half maximum (FWHM), $E_{F}=44 \mathrm{meV}$. The $E_{F}$ value obtained coincides pretty well with that found from the self-consistent solution of Schrödinger and Poisson equations $\left(E_{F}=43 \mathrm{meV}\right)$. The total electron sheet concentration, calculated from the simple relation $n_{S}=m_{e}^{*} E_{F} / \pi \hbar^{2}$ with the electron effective mass $m_{e}$ taken to be equal 0.066 [23], occurs to be equal $N_{s}=$ $1.21 \cdot 10^{12} \mathrm{~cm}^{-2}$, against the value $N_{s}=1.18 \cdot 10^{12} \mathrm{~cm}^{-2}$, calculated from Eqn. (1), and $n_{s}=1.15 \cdot 10^{12} \mathrm{~cm}^{-2}$, derived from the Shubnikov-de-Haas measurements. It should be mentioned that the low-energy luminescence threshold on the energy axis (see Fig. 2) is somewhat shifted towards the red side with respect to the $E_{11}$ peak position. This is a consequence of the $2 \mathrm{D}$-carrier scattering by the charge impurities, which strongly depends on the thickness of the spacer layer. The blue-shift of $E_{11}$ peak against the energy gap (by $7 \mathrm{meV}$ ) and the value of $\mathrm{G}$, observed experimentally, are exactly correspond to the $7.5 \mathrm{~nm}$ spacer in our samples. These findings give us ground to state that the PL profile is mainly defined by the electron-hole recombination accompanied by the impurity and disorder-assisted processes, resulting in the broadening and blueshift of the PL line. The spectral shift occurs due to subtraction of the intensity from the low-energy side of spectral profile and its addition to the high-energy region. The low-energy tail of the PL feature in Fig. 2 is approximated by the Urbach form.

The transformation of the PL line shape in the close vicinity of the $E_{F}$ under excitation density and/or temperature variation gives strong evidence for the FES manifestation, which was not accounted in foregoing analysis. Taking into account now the close proximity of $E_{F}$ and $E_{2}$ energies in our experiment a strong interaction between the elementary excitations near the Fermi level and the $n=2$ bare (atomic) exciton is expected. In general, the behavior of the FES and the $n=2$ exciton features is known [9-11,24] and follows the scenario: two features coexist in PL spectra, one is related to the FES and another is ascribed to the $n=2$ exciton. Temperature influences strongly the FES feature, due to low binding energy of the Mahan exciton. Typically the FES related enhancement in PL spectra disappears in the region of $10 \mathrm{~K}$. Xu et al. [11] observed the repelling between the FES and the $n=2$ exciton at low temperature and low excitation densities, resulting in the $n=2$ excitonic feature finally observed as a dominating feature in a PL spectrum at a higher intensity or temperature when the thermal population of the $n=2$ electron subband becomes of importance.

We have revealed now new peculiarities of the FES manifestation: starting from the FES origination up to its decay in PL. As one can see from PL spectra (Fig. 3) 
W.T. Masselink et al.: Pseudomorphic modulation-doped AIGaAs/InGaAs/GaAs heterostructures...

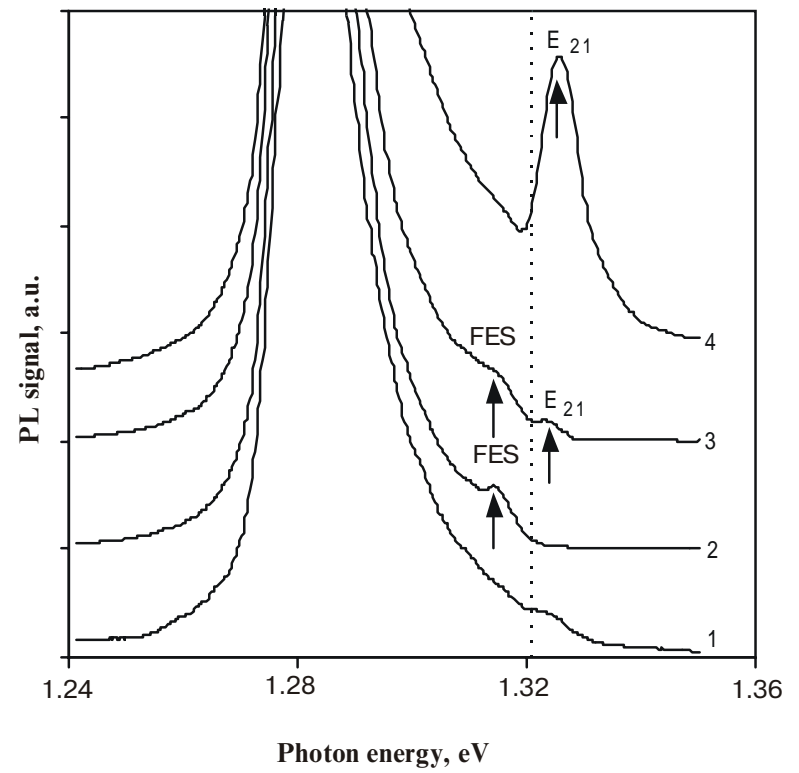

Fig. 3. Transformation of the high energy PL tail with intensity elevation. Curves 1 to 4 correspond to the intensities: $1-0.002 I_{0}$, $2-0.01 I_{0}, 3-0.1 I_{0}, 4-I_{0}$, respectively $\left(I_{0}=20 \mathrm{~W} / \mathrm{cm}^{2}\right)$. Transition energies for the FES and the $n=2$ exciton are shown by arrows up.

at the modest excitation level the PL tail spreads far beyond the $E_{F}$ boundary. No traces of the $n=2$ exciton exist. When the excitation density subsequently increases the FES peculiarity clearly develops with a prominent shift towards the energies below $E_{F}$. The steepness of the spectral drop-fall after the FES feature abruptly grows, and no PL signal is detected after $E_{F}$. Further increasing the excitation density gives the hint for the $n=2$ feature appearence, which moves towards a higher energy. Finally the $E_{11}$ and $E_{21}$ peaks are present alone at high intensities and the FES decays completely. Fig. 4 shows the motion of the FES and the $n=2$ related features under the intensity variation. One can easily see that the blueshift of the $n=2$ feature as well as the redshift of the FES feature tends to saturation. It is worthy to notice that the spectral shifts within the $E_{F}$ proximity are not accompanied by any noticeable shift of the $E_{11}$ transition if the excitation density increases. The blueshift of the $E_{21}$ peak is interpreted in terms of the transition from the hybridized exciton state to the bare (atomic) exciton [9$11,24]$, when the screening of the $n=2$ excition by excess electrons is included. Insensitivity of the $E_{11}$ transition to the pumping level gives evidence that the internal electrical field does not change under intensity increase, and therefore the blueshift saturation for the $E_{21}$ transition is mainly connected with the exciton screening and the transition from the excitonic recombinaton to the free-electron-hole recombination. This transition has been demonstrated by the change of the PL excitation spectra with excitation density in [11].

Typically the FES is interpreted as an enhancement of the oscillator strength for transitions close to the Fermi

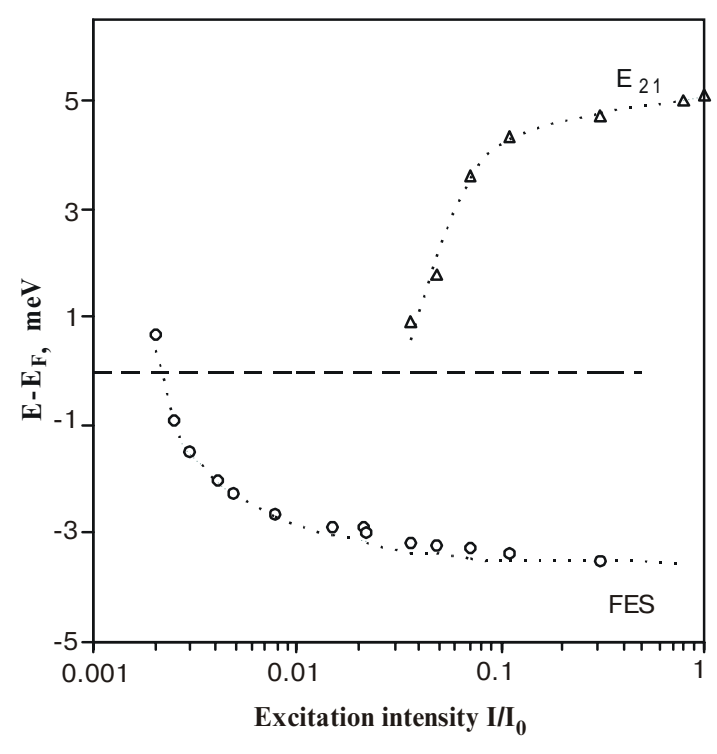

Fig. 4. Shift by energy for the FES and $n=2$ related features of the PL spectrum with the excitation density increase.

edge, when disorder or hole localization allows wavevector-nonconserving transitions. The optical oscillator strength for the Fermi level electrons annihilating with photoholes can be enhanced by over two orders of magnitude when the resonant coupling with the excitonic state is included [13]. The strength of this coupling depends strongly on the energy spacing between the states $\left(\Psi_{1}\right.$ and $\Psi_{2}$ ) involved in the resonance, the interaction matrix elements $V_{12}=\left\langle\Psi_{1}|V| \Psi_{2}\right\rangle$, and the optical matrix elements for the FES in the $n=1$ subband and the $n=2$ exciton transitions. In general, when the resonance coupling between discrete states is realized, two sets of hybridized states arise, and the oscillator strength becomes shared among them, the states of a smaller oscillator strength being typically contributed stronger by those of a larger oscillator strength. However, the resonance interaction affects strongly the density of states also, due to repulsion of the interacting quantum mechanical states. In our case, the quantum mechanical coupling between a discrete state and an energeticaly degenerate continuum of states takes place resulting in a Fano's interference. This interference manifests itself in the optical absorption spectrum by an asymmetric line shape, the so-called Fano-Beutler profile, which has the form [25]:

$\alpha \propto \frac{(\varepsilon+q)^{2}}{1+\varepsilon^{2}}$

where $\varepsilon$ is a normalized energy which is given by $\varepsilon=(\Omega$ $E) / \Gamma$ with $\Omega$ being the resonance energy of the discrete state and $\Gamma$ describing the strength of the coupling be- 


\section{W.T. Masselink et al.: Pseudomorphic modulation-doped AlGaAs/InGaAs/GaAs heterostructures...}

tween the discrete state and those of the continuum. $\Gamma$ is related to the coupling matrix element $V_{12}$ by $\Gamma=\pi\left|V_{12}\right|^{2}$. The parameter gives the ratio of the optical transition matrix elements for the transitions to the discrete state and to the continuum. The profile of Eq. (3) has a pronounced minimum at the energy when the interference between the transition amplitudes of the discrete state and the continuum is destructive. The reported experimental observations and theoretical predictions of Fano interference involve atomic systems, biexciton resonances, doped semiconductors and QW structures [26-28]. The Fano resonance aspect of interaction between an exciton and $2 \mathrm{D}$ electrons in particular $\mathrm{In}_{\mathrm{y}} \mathrm{Ga}_{1-\mathrm{y}} \mathrm{As}$ asymmetric single QW's was discussed by Chen et al. [10], without solving the many-body problem. The elaborated theoretical treatment including the band-gap renormalization, static screening, and dynamical response of the Fermi sea has been carried out by Hawrylak [13]. The predicted evolution of the absorption and emission spectra when the Fermi energy is varied over the range, wide in comparison with the exciton binding energy, is as follows: initially, the absorption and emission spectra are pinned to the Fermi level in the conduction band. With inclusion of the hole self-energy, the position of the actual emission line falls close to the bare excitonic transition, and in absorption two peaks arise, one being related to the bound exciton state. When carrier density increases the emission spectrum resembles the single-particle density-of-states in the conduction band with enhancement at the Fermi level, while the absorption spectrum evolves into a blueshifted FES. The behavior predicted is somewhat obscuring in comparison with that observed in experiment, including our data and those presented in [11].

Indeed, at first stage we observed the spread high energy tail of the PL feature (curve 1 in Fig. 3), which at low temperature and at low pumping level is reasonably described in terms of the electron-heavy hole recombination contributed by the impurity and disorder-assisted processes (Fig. 2). The FES enhancement, if exists, is of a negligible magnitude. When the excitation density is elevated in addition to the excitations of the Fermi sea, an increasing contribution from excitations including the holes in the $n=1$ heavy hole subband and the electrons in the second subband arises. Due to the strong resonance coupling of excitons with excitations of the Fermi sea two sets of hybridized states appear, separated by the region with destructive Fano interference. Those related to the Fermi edge are shifted by energy towards the low energy side, others related to the hybridized excitons go to the higher energies. Because of the modest excitation level these latter can not be observed in PL by the reason that the $n=2$ electron level occurs empty under the conditions considered. As a result in the PL spectrum (curve 2 in Fig. 3) we observe the typical FES enhancement with an abrupt fall-down from the high energy side, which signalizes about the Fano dip existence. Further increase of excitation density leads to the population of the $n=2$ electron level and the observation of the $E_{21}$ transition, which corresponds to the hybridized excitonic state yet (curve 3 in Fig. 3). Elevating the intensity we reach the level when both the FES and the excitonic emission become subsequently quenched and the free-2D electronhole recombination dominates at the high-energy PL tail (curve 4 Fig. 3).

Fig. 4 summarizes the behavior of the FES and the $n=2$ excitonic features with excitation density elevation. The region of intensities where only the FES originates is clearly seen. The strong and rapid redshift accompanies the FES appearence. This shift is practically saturated when the hybridized exciton state arises. The corresponding spectral feature demonstrates blueshift with a tendency to saturation, too, reflecting the screening of the $n=2$ exciton by excess electrons. In fact, we show here that the carrier concentration strongly influences the character of the FES manifestation. It is in line with conclusion derived from the PL investigation in the low-doped $\operatorname{In}_{\mathrm{x}} \mathrm{Ga}_{1-\mathrm{x}} \mathrm{As} / \mathrm{InP}$ heterostructures [12]. It was shown that in spite of the fact that both a close position of the Fermi edge to the empty $n=2$ electron subband and a hole localization by alloy fluctuation potential provide the optimal conditions for the occurrence of the FES, it occurs not sufficient for the FES enhancement to be developed, and the carrier concentration has to be adjusted also. We conclude that even the modest variation of the concentration, caused by the light illumination or heating, can substantially influence the FES development. Indeed, insignificant elevation of temperature (from $4.2 \mathrm{~K}$ to $15 \mathrm{~K}$ ) gives rise to a substantial broadening and subsequent smearing of the FES related feature in PL. The interpretation given above is consistent with the PL behavior observed, but we are of conscience that other models can be admitted for discussion.

The temperature elevation leads to a population of the higher electron and hole subbands and spreads the hole distribution in momentum space, allowing the direct elecron-hole recombination for more $2 \mathrm{D}$ electrons in the conduction band. In this case, a relation between the sheet carrier density and the energy separating the highenergy intensity cutoff and the intensity maximum of the first spectral band of the PL spectrum can be derived [29]. The PL linewidth at $T=77 \mathrm{~K}$ provides already a good measure of the sheet carrier density [30], and a correlation exists even between the sheet carrier density and the PL peak width for the $0_{\mathrm{e}}{ }^{2} \rightarrow 0_{\mathrm{h}}$ transition $[31,32]$. Fig. 5 shows the PL spectrum at $T=77 \mathrm{~K}$ and its modification with the excitation density varied within two orders of magnitude. The intensity of the single PL peak (cf. Fig. 2 ) is shared now between two transitions, $E_{11}$ and $E_{21}$. Both PL features become broadened, and the ratio of their maximums correlates with the calculated relative electron-hole function overlap for the $E_{11}$ and $E_{21}$ transitions in our self-consistent analysis. Fig. 6 demonstrates the change of the intensity ratio with temperature elevation determined experimentally, using two transitions involved, together with the calculated ratio for a given difference $E_{2}-E_{F}$. The intensity ratio tends the saturation at higher temperature due to the electron and hole Fermi distributions, smoothening and the recombination 
W.T. Masselink et al.: Pseudomorphic modulation-doped AIGaAs/InGaAs/GaAs heterostructures...

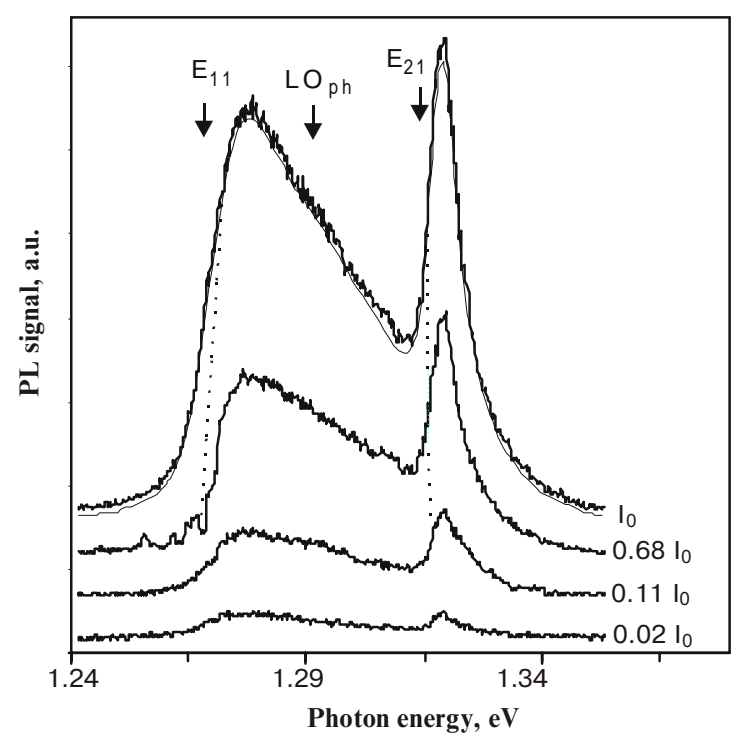

Fig. 5. PL spectrum at $77 \mathrm{~K}$ under elevation of the excitation density. Fit for both $E_{11}$ and $E_{21}$ transitions to Eq. 2 is shown by dashed line. Arrows mark the calculated $E_{11}$ and $E_{21}$ transition energy and the LO-phonon replica of 2DEG.

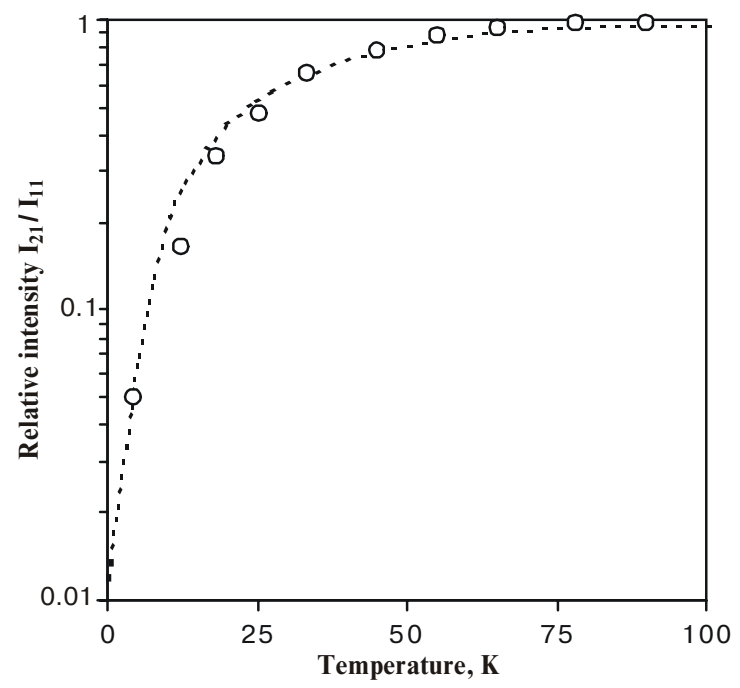

Fig. 6. Temperature-related change of the experimental ratio of PL intensities for the $E_{11}$ and $E_{21}$ transitions. Dashed line is the calculated dependence for $E_{2}-E_{F}=5 \mathrm{meV}$.

probabilities become equalized for different $k$-values. The fit of the PL spectrum, shown in Fig. 5 by dashed line, is performed using Eq. 2, where both $E_{11}$ and $E_{21}$ transitions are taken into account. The broadening parameter, which characterizes phenomenologically the carrier-ionized impurities scattering, is assumed to be the same for both peaks. We have found, following Gilperez et al. [23], that the effect of temperature-assisted shift of the peak maxima with respect to the $k=0$ transition energies can not be ruled out and has to be added to the shift caused by Coulombic interactions when the carrier-ionized impurities scattering is considered in terms of the Lyo and Jones theory [22]. The shoulder seen between two PL peaks (Fig. 5) is interpreted as LO-phonon replica in the relaxation of hot electrons at high densities. These GaAslike LO phonons participate in the scattering processes of the electrons in the Fermi sea. The shoulder observed becomes even more pronounced with the temperature elevation, providing evidence of its phonon nature. The results of the fit within the Lyo and Jones theory were compared also with those found from a phenomenological line-shape model developed by Brierley [32]. From the Fermi level and the two electron subband energies the channel sheet density was determined using Eq. 1. The $N_{s}$ values, derived from both theories, coincides within the $7 \%$ accuracy and found to be $\left.N_{S}=1.16 \pm 0.05\right) \cdot 10^{12}$ $\mathrm{cm}^{-2}$ versus that of $N_{s}=1.21 \cdot 10^{12} \mathrm{~cm}^{-2}$ found from the low temperature fit. The results obtained show that our assessment for pseudomorphic modulation-doped $\mathrm{Al}_{\mathrm{x}} \mathrm{Ga}_{1-\mathrm{x}} \mathrm{As} /$ $\mathrm{In}_{\mathrm{y}} \mathrm{Ga}_{1-\mathrm{y}} \mathrm{As} / \mathrm{GaAs}$ heterostructures agrees good with that derived from the self-consistent description, based on the solution of the coupled Schrödinger and Poisson equations.

\section{B. PL in magnetic field}

Free electrons and holes are quantized into Landau levels (LL) when a perpendicular magnetic field is applied. We have shown [8] by means of optical detection of quantum oscillations in PL that two sorts of holes are present in our QW heterostructures: nearly free, or localized in shallow traps caused by fluctuations of potential, and those which are strongly localized by alloy fluctuations (with the localization energy of order of $10 \mathrm{meV}$ ). These latter holes are probably responsible for the FES enhancement found. In magnetic field the PL spectrum breaks up into a series corresponding to transitions between the LL's of the heavy hole valence band, signified by the hole Landau level number $N_{h}$, and those of the electron conduction band, labeled by $N_{e}$. Electron-hole recombinations occur between the LL with the same quantum numbers $\left(N_{e}-N_{h}=0\right)$, according to a well-known selection rule [33]. Under the low excitation density and at low temperature, the most of the photoexcited holes relax to the ground-state $\mathrm{LL}, 0_{h}$, and the radiative transition with $N_{e}=0$ is allowed only, while the transitions with $N_{e}>0$ are nominally forbidden. Due to the carrier-impurity interactions these indirect transitions become allowed and give the main contribution to the magnetoluminescence. The commensurate LL spectral structure is clearly seen in our samples (Fig. 7). The electron-hole emission arising primarily from the direct $0_{e} \rightarrow 0_{h}$ transitions is accompanied by the emissions from indirect $1_{e} \rightarrow 0_{h}, 2_{e} \rightarrow$ $0_{h}, 3_{e} \rightarrow 0_{h}$, etc., transitions, which are generally higherorder effects and have much weaker oscillator strengths when compared with the diagonal transitions $\left(N_{e}-N_{h}=\right.$ $0)$. With increasing magnetic field the successive LL depopulate due to the inter-LL separation $\hbar \omega_{c}=\hbar e B / m_{e}^{*}$ and the LL degeneracy ( $2 e B / h$ including spin) increase 


\section{W.T. Masselink et al.: Pseudomorphic modulation-doped AlGaAs/InGaAs/GaAs heterostructures...}

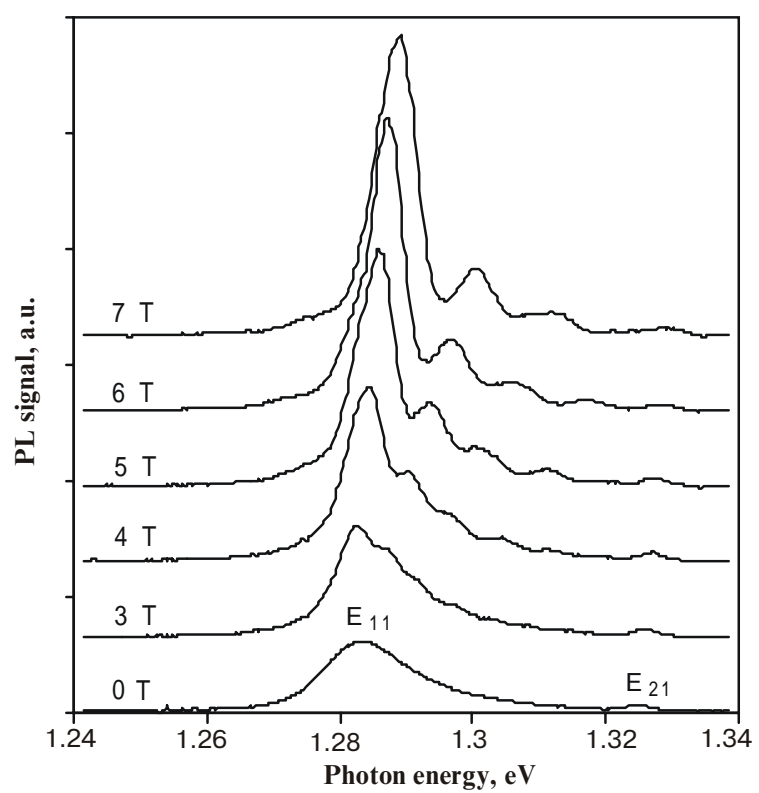

Fig. 7. Low temperature PL spectrum under variation of magnetic field $(T=6 \mathrm{~K}) . E_{11}$ and $E_{21}$ transition energies at $B=0 \mathrm{~T}$ are marked by arrows.

with field, and at $B=7 \mathrm{~T}$ only $0_{e} \rightarrow 0_{h}, 1_{e} \rightarrow 0_{h}, 2_{e} \rightarrow 0_{h}$ transitions are clearly resolved. The $n=2$ PL feature is present at each magnetic field strength. The variation of the transition energies with magnetic field is summarized in Fig. 8. The even LL filling factor $v=h N_{S} / e B$ including spin is shown by vertical bars. The energy separation corresponds to the electron cyclotron frequency. The shift rate of the $0_{e} \rightarrow 0_{h}$ transition is equal to one half of the

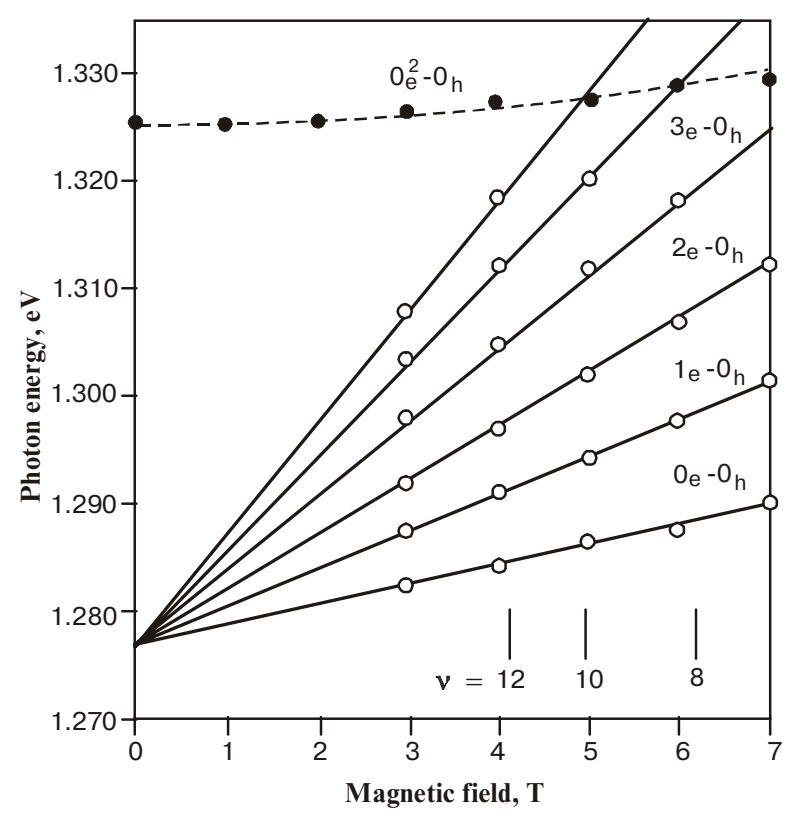

Fig. 8. Transition energies of LL versus magnetic field. The shift of the $n=2$ related feature is approximated by $B^{2}$ dependencedashed line. Filling factor is shown by vertical bars.
inter-LL splitting. The onset of the $n=1$ electron subband extrapolated from the LL transitions is found to be $1.277 \mathrm{eV}$, coinciding close with that found from the PL line shape fit. The slope of lines extrapolating the experimental data in Fig. 8 is immediately connected with the effective masses of carriers involved in transition by

$$
E(B)-E(0)=B e \hbar\left[\frac{1}{2 \mu}+\frac{N_{e}}{m_{e}^{*}}+\frac{N_{h}}{m_{h}^{*}}\right],
$$

where $\frac{1}{\mu}=\frac{1}{m_{e}^{*}}+\frac{1}{m_{h}^{*}}$ is the reduced mass. The electron effective mass is derived to be of $m_{e} *=(0.068 \pm 0.003) m_{0}$ ( $m_{0}$ is the free electron mass), close to the value determined by Butov et al. [34], but typically higher of that determined from the Shubnikov-de-Haas measurements. The heavy hole mass is found to be $m_{h}{ }^{*}=(0.150 \pm$ $0.003) m_{0}$. The intensity of the $n=2$ excitonic emission is expected to be strongly influenced when a magnetic field is applied. Indeed, we observed the growth of the PL signal in this spectral position, when magnetic field is scanned up to $7 \mathrm{~T}$. The observed shift of the peak position is proportional to $B^{2}$, which is a characteristic dependence for diamagnetic shift of the excitonic transitions. In analog to the zero-field case, the photoholes, occupying the lowest LL are actual in transitions at low temperature, resulting in the off-diagonal transitions even stronger than the diagonal ones in our magnetoluminescence measurements. The strength of the off-diagonal transitions depends eventually on the thickness of the spacer layer, resulting in more pronounced transitions for thinner spacer layer [10]. The transitions up to $N_{e}=6$ are surely detectable in magneto-PL at low temperatures. The behavior of the transition energies $N_{e} \rightarrow 0_{h}$ observed is typical of a $2 \mathrm{DEG}$ with a single filled subband, besides the region of the $n=2$ transitions. They increase with $B$ due to an increase of the electron cyclotron energies and oscillate weakly near the position of the corresponding LL energies. This latter effect is connected with oscillations of the exchange and correlation energies [35]. The behavior of the PL feature corresponding to the $n=2$ transitions is strongly influenced each time when the successive LL approach. As a result we observed a considerable increase of the PL magnitude when the last $3_{e} \rightarrow 0_{h}$ transition (from the series of LL which crossover the $n=2$ level at $B=7 \mathrm{~T}$ ) occurs close to the energy of the $n=2$ excitonic transition. The spectral shape of PL resembles the plateau within $12 \mathrm{meV}$ region with abrupt fall-down at the high-energy side. The Coulomb field from the $0_{h}$ hole state induces the admixture of the $0_{e}{ }^{2}$ state to the LL state, resulting in their enhancement because the oscillator strength for the allowed $0_{e}^{2} \rightarrow 0_{h}$ transition is more than two orders of magnitude larger [10]. In fact, the plateau is formed also for the $2_{e} \rightarrow 0_{h}$ transition and the appearance of these plateaus gives evidence of complicated structure of the corresponding PL features, non-resolvable at the available magnetic field up to $7 \mathrm{~T}$. We expect that at higher magnetic field the complicated interplay of the magnetoexciton states, $0_{e}{ }^{2} \rightarrow 0_{h}$ and $N_{e} \rightarrow 1_{h}$ might develop [36]. 


\section{W.T. Masselink et al.: Pseudomorphic modulation-doped AlGaAs/InGaAs/GaAs heterostructures...}

In part the overlapping of different contributions in the region of plateaus is lifted off by the temperature increase. The temperature dependence of diagonal and offdiagonal transitions is principally different. The thermal populations of the higher hole LL grow under temperature elevating. As a result the intensity of diagonal transitions increases and they dominate the PL spectra at high temperature. Such a temperature induced evolution of the PL spectrum is shown in Fig. 9. It can be seen that the $0_{e} \rightarrow 0_{h}$ and $1_{e} \rightarrow 0_{h}$ transitions progressively decrease by intensity with the temperature increase, whereas the $2_{e} \rightarrow 0_{h}$ feature demonstrates obviously splitting already at $T=12 \mathrm{~K}$ with rapid temperature quenching of the high energy component. The low-energy component grows subsequently and has the magnitude equal to that of the $1_{e} \rightarrow 0_{h}$ transition at $T=45 \mathrm{~K}$. This feature is unequivocally connected with the $1_{e} \rightarrow 1_{h}$ transition. Its magnitude follows the Boltzmann thermal distribution for the hole level. Therefore, the low temperature plateau at the $2_{e} \rightarrow 0_{h}$ PL feature at $B=7 \mathrm{~T}$ is obviously formed with participation of the $1_{e} \rightarrow 1_{h}$ transition. The $2_{e} \rightarrow 2_{h}$ transitions have to contribute the plateau observed at low temperature in the region of the $3_{e} \rightarrow 0_{h}$ off-diagonal transitions. The $2_{e} \rightarrow 2_{h}$ transition becomes well pronounced in this region at temperature as high as $45 \mathrm{~K}$. Fig. 10 shows the temperature shift of the emission lines for the inter LL transitions in magnetic field $B=7$ $\mathrm{T}$. The corresponding change of the band-gap energy is

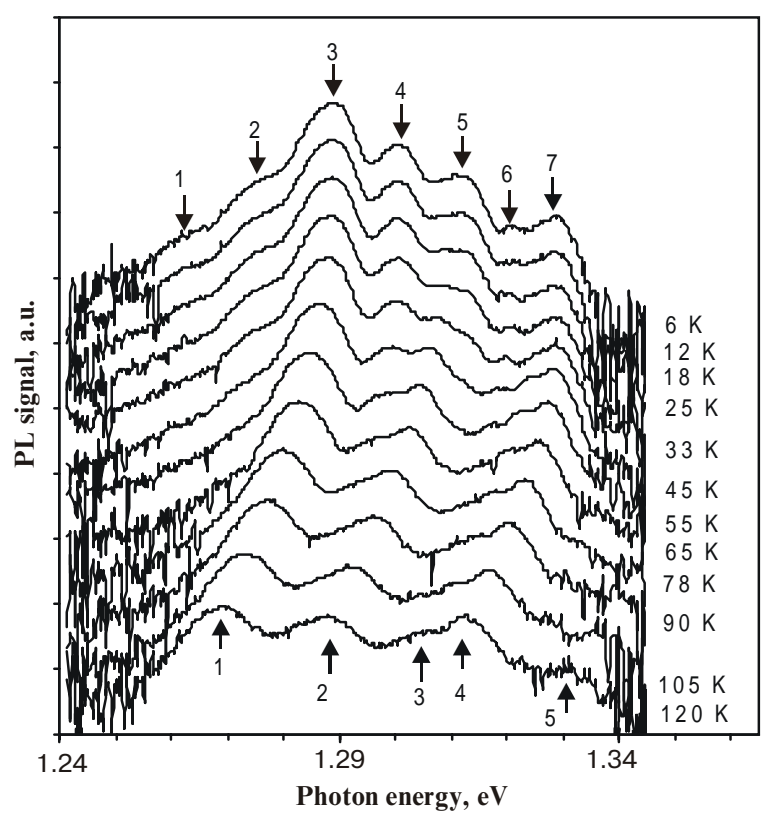

Fig. 9. Temperature related evolution of the PL spectrum in a magnetic field $(B=7 \mathrm{~T})$. Arrows down mark: $1-1_{e} \rightarrow 0_{h} \mathrm{LO}$ phonon side band, $2-2_{e} \rightarrow 0_{h}$ phonon side band, $3-0_{e} \rightarrow 0_{h}$ transition, $4-1_{e} \rightarrow 0_{h}$ transition, $5-2_{e} \rightarrow 0_{h}$ transition, 6 - FES cusp, $7-0_{e}{ }^{2} \rightarrow 0_{h}$ transition. Arrows up stand for: $1-0_{e} \rightarrow 0_{h}$ transition, $2-1_{e} \rightarrow 1_{h}$ transition, $3-2_{e} \rightarrow 2_{h}$ transition, $4-0_{e}{ }^{2} \rightarrow 0_{h}$ transition, and 5 - LL transitions related to $n=2$ subband.

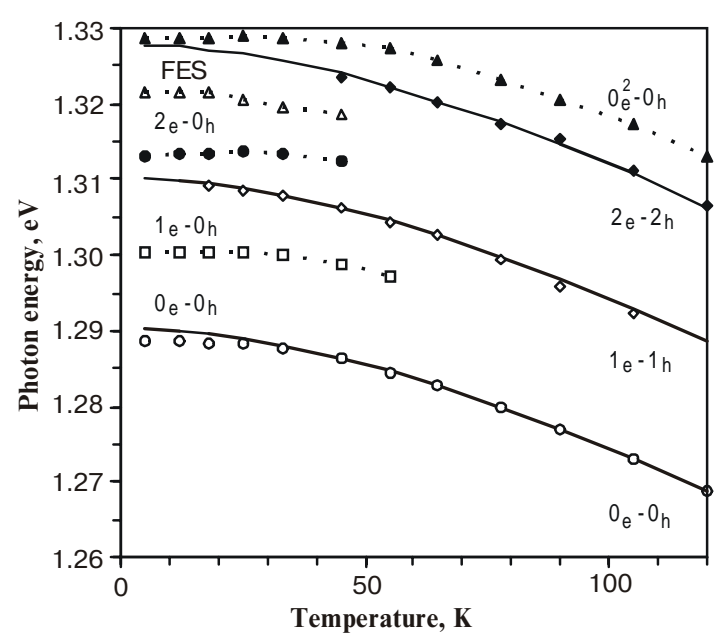

Fig. 10. Temperature dependence of the inter LL transition energies. The solid lines show the temperature change of the band gap.

shown by the solid line. The redshift of the entire spectrum is due to the band-gap shrinkage, while the LL spacings remain intact. There exists a well pronounced deviation of the experimental points from the line reflecting the temperature dependence of the band-gap. This deviation observed at low temperature is immediately connected with fluctuations of the local potential energy in the quantum well. According to Butov et al. [34] at low excitation densities and low temperatures, the photoexcited holes occupy predominantly the low-energy states within the broadened Landau levels, and the transition energy is smaller than the gap between the corresponding electron and hole LL. The width of the $n$-th LL, $\Gamma_{n}$, is determined by both magnitude of the fluctuations and their sizes. The magnetic length at $B=7 \mathrm{~T}$ occurs of the same order as a characteristic scale of the potential fluctuations in our quantum wells. In this case [37] the LL width decreases with increasing the number $N_{e}$. An increase of temperature results in more regular distribution of holes over all states at LL and the inter LL transition energies follow the temperature variation of $E_{g}$. The similar effect is expected under the excitation density elevation.

Fig. 11 shows the PL spectrum evolution when the pumping density grows. It is clearly seen that the $1_{e} \rightarrow 1_{h}$ related feature appears under the intensity increase by one order of magnitude. This appearance can not be explained by subsequent filling of the hole LL. Butov et al. [34] assumed that the 'excess' recombination involving excited hole states arises due to increase of the hole relaxation rate with the hole concentration increasing. At low excitation level the defect-induced energy relaxation of the holes in quantum well is a dominant mechanism of photoexcited hole relaxation. An increase in the electron-hole temperature when the hole concentration is small enough, $n_{h}<<n_{s}$ could give evidence for the effective suppression of this channel of hole relaxation by the photoexcited holes which neutralize the large-scale fluctuation.

SQO, 3(2), 2000 
W.T. Masselink et al.: Pseudomorphic modulation-doped AlGaAs/InGaAs/GaAs heterostructures...

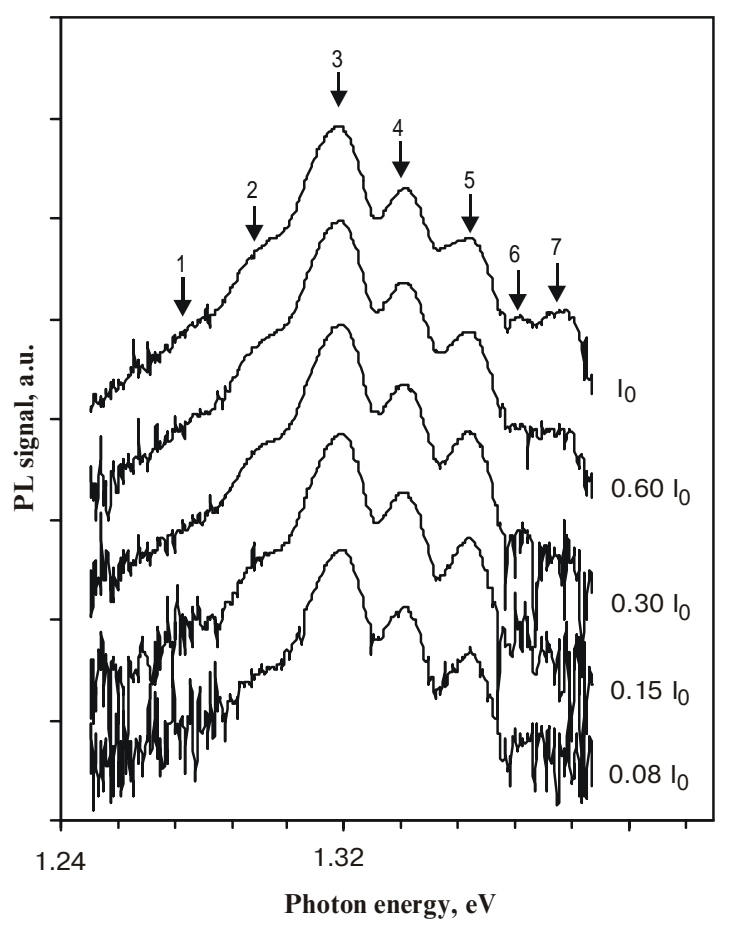

Fig. 11. Excitation density related evolution of the PL spectrum in a magnetic field $(B=7 \mathrm{~T})$ and at low temperature $(T=6 \mathrm{~K})$. Arrows down have the same meaning as in Fig. $9\left(I_{0}=20 \mathrm{~W} / \mathrm{cm}^{2}\right)$.

New features of interest arise at the low energy side of the PL spectrum in magnetic field $B=7 \mathrm{~T}$ when the excitation density is elevated. No features were detected when magnetic field is not applied. The physical reasons for such feature development in a magnetic field have been widely discussed [38-41]. Both LO-phonon and interLL shake up excitations were reported in the magneto PL spectra of modulation-doped pseudomorphic $\mathrm{Al}_{\mathrm{x}} \mathrm{Ga}_{1-\mathrm{x}} \mathrm{As} / \mathrm{In}_{\mathrm{y}} \mathrm{Ga}_{1-\mathrm{y}} \mathrm{As} / \mathrm{GaAs}$ quantum well structures with high carrier density [38]. The $\Delta N_{e}=1$ and $\Delta N_{e}=2$ shake-up satellites were observed at energies close to the predictions of the many-body theory for magneto-plasmon excitations in a high-density system. The importance of hole localization as well as screening in determining the strength of the carrier-LO-phonon interaction has been demonstrated [40]. Typically for guaranteed observation of the shake-up satellites below the principal $0_{e} \rightarrow 0_{h}$ transition in magnetic field the magnetic field strength has to be a substantially higher that available. Therefore, we incline to consider the features observed at the elevated excitation density to be the LO-phonon side bands described by Lyo et al. [41]. The LO-phonon side bands for the transitions between the electron and hole LL are relatively strong and grow with the $N_{e}$ number. Typically the side-bands with a smaller $N_{e}$ are substantially weaker, and the side-band which accompanies the primary $0_{e} \rightarrow$ $0_{h}$ transition in $n$-GaAs/InGaAs/AlGaAs QW is absent at low temperature in the magnetic field as large as $B=9 \mathrm{~T}$. Lyo et al. [41] have shown that the $0_{e} \rightarrow 0_{h}$ related LO side-band begins to be visible above $B=15 \mathrm{~T}$ because $m_{h} *$ increases with the energy of the $0_{h}$ heavy hole level due to the valence band nonparabolicity. The intensity peaks of the phonon side-bands are inverted in relative strength compared with those of the zero-phonon lines. This effect is caused by the suppression of small $\Delta N=\left(\left|N_{e}-N_{h}\right|\right)$ side bands arising from the interference between the electron-phonon and hole-phonon recombination channels and the magnetic quantization in 2D system [41].

Following our assignment the PL features at $12 \mathrm{meV}$ and at $23 \mathrm{meV}$ below the $0_{e} \rightarrow 0_{h}$ transition are the LOphonon side bands for the $2_{e} \rightarrow 0_{h}$ and $1_{e} \rightarrow 0_{h}$ LL transitions, respectively, with the well-known GaAs LOphonon energy $36.2 \mathrm{meV}$. It is interesting that the features observed are practically hidden at the noise level when the excitation density is small enough and become strongly enhanced with the intensity elevation. Finally, they reach the magnitudes comparable with those of the off-diagonal parent transitions. Such strong enhancement of the LO-phonon side bands as well as their evolution with the excitation density increase are, to best of our knowledge observed for the first time. Another possible source of feature appearance might be the holes deeply localized by potential fluctuations (localization energy $\sim 10 \mathrm{meV}$ ) [8]. However, the absence of these features at zero magnetic field under variation of excitation density by three orders of magnitude makes this possibility likely insignificant.

The high energy side of the magneto PL spectrum (Fig. 11) undergoes the radical transformation also with the excitation density increase. It is obviously seen the coexistence of two spectral features, at least. The high energy one is unambiguously related to the $1_{e} \rightarrow 1_{h}$ transition. The low energy hump could be ascribed to the $2_{e} \rightarrow 1_{h}$ transition, however this latter becomes normally visible at higher excitation level, when the $1_{e} \rightarrow 1_{h}$ becomes one of the dominating features in the PL spectrum [34]. In our experiment the hump is well pronounced already far before the hint for the $1_{e} \rightarrow 1_{h}$ transition appears. In spectral evolution with excitation density we observe repelling this feature with that of the $1_{e} \rightarrow 1_{h}$ transition. Thorough examination of the transformation in this spectral region with excitation density variation shows a remarkable resemblance with that observed without magnetic field in the proximity of Fermi edge. Indeed, in a perpendicular magnetic field the $2 \mathrm{D}$ electron density of states is quantized and the Fermi level sweeps within the successive LL. As a result the periodical (in $1 / B$ ) tuning of the Fano resonance occurs for the FES and $n=2$ exciton state. The pronounced enhancement of the FES can be realized when at least two conditions are fulfilled: $E_{F}$ energy falls down within the extended states; the resonant coupling originates between the $n=2$ exciton and the nearest interacting LL [3]. Both of these conditions are kept in our case and the observation of the resonant FES enhancement in magnetic field mirrored by hump, is actual. Therefore, the hump is assumed to be here a manifestation of many body effects, developed in the vicinity of the Fermi energy in magnetic field. 
The comparison of Figs 9 and 11 shows that both low and high energy sides of the PL spectrum in magnetic field substantially evolve under temperature elevation. Besides a total redshift of the spectrum the LO-phonon side bands subsequently decay, the low energy $1_{e} \rightarrow 0_{h}$ phonon side disappears when temperature reaches $T=25 \mathrm{~K}$, while the $2_{e} \rightarrow 0_{h}$ phonon side is still visible at $T \cong 50 \mathrm{~K}$, giving evidence of stronger coupling LOphonon with the $2_{e} \rightarrow 0_{h}$ transition. The hump, related to many body effects is surely detectable even at $T \cong 55 \mathrm{~K}$. The energy separation between its spectral position and that of the $0_{e}{ }^{2} \rightarrow 0_{h}$ is approximately invariable. When temperature is elevated up to $T \geq 100 \mathrm{~K}$, the LL structure arises, connected with the $n=2$ electron subband. The findings demonstrated here are not striking. Indeed, numerical calculations [16] have shown the electron-electron interaction in the degenerate Fermi sea strongly correlate excitons and the Fermi edge transitions can survive for the first electron subband, at least, for temperatures $T \sim 50 \mathrm{~K}$. Recent studies of magneto-optical Kerr-ellipticity with different InGaAs/InP multiple-quantum-wells at room temperature have shown that the excitonic and Landau states can coexist even at room temperature [42].

\section{Conclusions}

Summarizing the results of PL study in the pseudomorphic modulation-doped $\mathrm{Al}_{\mathrm{x}} \mathrm{Ga}_{1-\mathrm{x}} \mathrm{As} / \mathrm{In}_{\mathrm{y}} \mathrm{Ga}_{1-\mathrm{y}} \mathrm{As} / \mathrm{GaAs}$ heterostructures, possessing the high electron density, we observed a fundamental changes of the PL spectrum at temperature elevation and excitation density increase. In most the high and low energy tails of the PL feature undergo the principal transformations. High-energy tail peculiarities are related to the repelling of the FES and the excitonic states. The character of repelling depends crucially on the excitation density and temperature. At low temperature the origination of the FES feature has been observed for the first time under increasing the excitation density. The FES appearance accompanies by the formation of an abrupt high energy edge and occurs far below by intensity the hybridized $n=2$ exciton manifestation. Strong screening of the $n=2$ exciton state by photoexcited carriers is observed, resulting in the 2DEGheavy hole recombination for second electron subband. The many-body feature is detected in the magnetoluminescence spectrum, possessing the hump-shape. This feature develops in magnetic field $B=7 \mathrm{~T}$ at low temperature $(T=4.2 \mathrm{~K})$ and is surely detected up to $T \approx 50 \mathrm{~K}$. The LO-phonon side bands for parent transitions between the LL are revealed in the low-energy tail of the PL spectra in magnetic field. The evolution of these phonon sideband with temperature and excitation density is observed. A strong excitation power dependence of the 2D-related PL could be the result of the photoinduced change of the potential distribution across the quantum well structure, as has been previously observed in $\mathrm{Al}_{\mathrm{x}} \mathrm{Ga}_{1-\mathrm{x}} \mathrm{As} / \mathrm{GaAs}$ quantum structures [43,44]. A strong band bending might arise from the neutralization of the ionized residual ac- ceptors by photocreated holes in the $\mathrm{In}_{\mathrm{y}} \mathrm{Ga}_{1-\mathrm{y}} \mathrm{As}$ channel and the neutralization of the ionized donors in the barriers by photocreated electrons. The reduction of the band bending under light illumination leads to typical blueshift of the PL spectrum. We have not observed a noticeable blueshift of the PL spectrum and hence this reason has to be ruled out. We incline to explain the behavior of the PL near the Fermi edge by a strong carrier density effect on the FES manifestation not yet explored theoretically.

\section{Acknowledgments}

This work is supported by NATO linkage grant.

\section{References}

1. Schaff W. J., Tasker P. J., Foisy M. C., and Eastman L. F. Strained layer superlattices: Materials science and technology // Semiconductors and Semimetals 33, Ed. Pearsall T., pp. 73-138, New York: Academic Press (1991).

2. Henderson T., Aksun M. I., Peng C. K., Morcoç H., Chao P. C., Smith P. M., Duh K. H. G., and Lester L. F. Microwave performance of a quarter-micrometer gate low-noise pseudomorphic InGaAs/AlGaAs modulation-doped field effect transistor // IEEE Electron. Device Lett. 7(12), pp. 649651 (1986).

3. Chen W., Fritze M., Nurmikko A. V., Ackley D., Colvard C., and Lee H. Interaction of magnetoexcitons and twodimensional electron gas in the quantum Hall regime // Phys. Rev. Lett. 64(20), pp. 2434-2437 (1990).

4. Delalande C., Bastard G., Orgonasi J., Brum J. A., Liu H. W., Voos M., Weimann G., and Schlapp W. Many-body effects in a modulation-doped semiconductor quantum well // Phys. Rev. Lett. 59(23), pp. 2690-2692 (1987).

5. Skolnick M. S., Rorison J. M., Nash K. J., Mowbray D. J., Tapster P. R., Bass S. J., and A. D. Pitt Observation of a many-body edge singularity in quantum-well luminescence spectra // Phys.Rev. Lett. 58(20), pp. 2130-2133 (1987).

6. Chang Y. C. and Sanders G. D. Band-mixing effect on the emission spectrum of modulation-doped semiconductor quantum wells // Phys. Rev. B. 32(8), pp. 5521-5524 (1985).

7. Heiman D., Goldberg B. B., Pinczuk A., Tu C. W., Gossard A. C., and English J. H. Optical anomalies of the two-dimensional electron gas in the extreme magnetic quantum limit // Phys. Rev. Lett. 61(5), pp. 605-608 (1988).

8. Tarasov G. G., Müller U., Mazur Yu. I., Kissel H., Zhuchenko Z. Ya., Walther C., and Masselink W. T. Direct investigation of localized hole states in pseudomorphic modulation-doped $\mathrm{Al}_{\mathrm{x}} \mathrm{Ga}_{1-\mathrm{x}} \mathrm{As} / \mathrm{In}_{\mathrm{y}} \mathrm{Ga}_{1-\mathrm{y}} \mathrm{As} / \mathrm{GaAs}$ heterostructures by optical detection of quantum oscillations // Phys. Rev. B. 58(8), pp. 4733-4739 (1998).

9. Fisher T. A., Simmonds P. E., Skolnick M. S., Martin A. D., and Smith R. S. Fermi-energy edge singularity and excitonic enhancement associated with the second subband in asymmetric modulation-doped quantum wells // Phys. Rev. B. 48(19), pp. 14253-14263 (1993).

10. Chen W., Fritze M., Walecki W., and Nurmikko A. V. Excitonic enhancement of the Fermi-edge singularity in a dense two-dimensional electron gas // Phys. Rev. B. 45(15), pp. 8464-8477 (1992).

11. Xu S. J., Chua S. J., Tang X. H., and Zhang X. H. Strong interaction of Fermi-edge singularity and exciton related to $N=2$ subband in a modulation-doped $\mathrm{Al}_{\mathrm{x}} \mathrm{Ga}_{1-\mathrm{x}} \mathrm{As} / \mathrm{In}_{\mathrm{y}} \mathrm{Ga}_{1}$ yAs/GaAs quantum well // Phys. Rev. B. 54(24), pp. 1770117704 (1996). 


\section{W.T. Masselink et al.: Pseudomorphic modulation-doped AlGaAs/InGaAs/GaAs heterostructures...}

12. Buyanova I. A., Lundström T., Buyanov A. V., Chen W. M., Bi W. G., and Tu C. W. Strong effects of carrier concentration on the Fermi-edge singularity in modulation-doped InP/ $\mathrm{In}_{\mathrm{x}} \mathrm{Ga}_{1-\mathrm{x}}$ As heterostructures // Phys. Rev. B. 55(11), pp. 70527058 (1997).

13. Hawrylak P. Optical hole in a two-dimensional electron gas // Phys. Rev. B. 42(14), pp. 8986-8990 (1990).

14. Mueller J. F., Ruckenstein A., and Schmitt-Rink S. Interference of the Fermi edge singularity with an excitonic resonance in doped semiconductors // Mod. Phys. Lett. B. 5(2), pp. 135-138 (1991).

15. Livescu G., Miller D. A. B., Chemla D. S., Ramaswamy M., Chang T. Y., Sauer N., Gossard A. C., and English J. H. Free carrier and many-body effects in absorption spectra of modulation-doped quantum wells // IEEE J. Quantum Electron. 24(8), pp. 1677-1689 (1988).

16. Gumbs G., Huang D., and Fessatidis V. Many-body effects on temperature dependence of the interband absorption in quantum wells // J. Appl. Phys.75(12), pp. $7942-7948$ (1994).

17. Kissel H., Müller U., Walther C., Masselink W. T., Mazur Yu. I., Tarasov G. G., Zhuchenko Z. Ya. Peculiarities of photoluminescence in pseudomorphic modulation-doped $\mathrm{Al}_{0.2} \mathrm{Ga}_{0.8} \mathrm{As} / \mathrm{In}_{0.1} \mathrm{Ga}_{0.9} \mathrm{As} / \mathrm{GaAs}$ quantum wells // Phys. Rev. B. 58(8), pp. 4754-4760 (1998).

18. Guzzi M. and Steahly J. L. Physics of DX centers in III-V ternary compounds / Ed. Bourgoin J. C., Aedermannsdorf: Trans Tech. (1989).

19. Goetz K.-H., Bimberg D., Jürgensen H., Selders J., Solomonov A. V., Glinskii G. F., and Razeghy M. Optical and crystallographic properties and impurity incorporation of $\mathrm{Ga}_{\mathrm{x}} \mathrm{In}_{1-\mathrm{x}}$ As $(0.44<\mathrm{x}<0.49)$ grown by liquid phase epitaxy, vapor phase epitaxy, and metal organic chemical vapor deposition // J. Appl. Phys. 54(8), pp. 4543-4552 (1983).

20. Joyce M. J., Johnson M. J., Gal M., and Usher B. F. Concentration-dependent band offset in $\operatorname{In}_{\mathrm{x}} \mathrm{Ga}_{1-\mathrm{x}} \mathrm{As} / \mathrm{GaAs}$ strained quantum wells // Phys. Rev. B. 38(15), pp. 1097810980 (1988).

21. Hedin L. and Lundqvist B. I. Explicit local exchange-correlation potentials // J. Phys. C : Solid St. Phys. 4, pp. 2064 2083 (1971).

22. Lyo S. K. and Jones E. D. Photoluminescence line shape in degenerate semiconductor quantum wells // Phys. Rev. B. 38(6), pp. 4113-4119 (1988).

23. Gilpérez J. M., Sánchez-Rojas J. L., Mucoz E., Calleja E., David J. P. R., Reddy M., Hill G., Sánchez-Dehesa J. Roomand low-temperature assessment of pseudomorphic AlGaAs/ InGaAs/GaAs high-electron-mobility transistor structures by photoluminescence spectroscopy // J. Appl. Phys. 76(10), pp 5931-5944 (1994)

24. Colvard C., Nouri N., Lee H., and Ackley D. Optical investigations of the high-density electron gas in pseudomorphic $\mathrm{In}_{\mathrm{x}} \mathrm{Ga}_{1-\mathrm{x}} \mathrm{As}$ quantum-well structures // Phys. Rev. B. 39(11), pp. 8033-8036 (1989).

25. Fano U. Effects of configuration interaction on intensities and phase shifts // Phys. Rev. 124(6), pp. 1866-1878 (1961).

26. Glutsch S., Siegner U., Mycek M.-A., and Chemla D. S Fano resonances due to coupled magnetoexciton and continuum states in bulk semiconductors // Phys. Rev. B. 50(23), pp. - 17009-17017 (1994)

27. Oberli D. Y., Böhm G., and Weimann G. Fano resonances in the excitation spectra of semiconductor quantum wells // Phys. Rev. B. 49(8), pp. 5757-5760 (1994).

28. Cohen G., Shtrikman H., and Bar-Joseph I. Optical spectroscopy of Fano interference in a GaAs/ $\mathrm{Al}_{\mathrm{x}} \mathrm{Ga}_{1-\mathrm{x}} \mathrm{As}$ superlattice in a magnetic field // Phys. Rev. B. 52(16), pp. $11642-11645$ (1995)
29. Brugger H., Müssig H., Wölk C., Kern K., and Heitmann D. Optical determination of carrier density in pseudomorphic $\mathrm{AlGaAs} / \mathrm{InGaAs} / \mathrm{GaAs}$ hetero-field- effect transistor structures by photoluminescence // Appl. Phys. Lett. 59(21), pp. 2739-2741 (1991).

30. Dodabalapur A., Kesan V. P., Hinson D. R., Neikirk D. P., and Streetman B. G. Photoluminescence studies of pseudomorphic modulation-doped AlGaAs/InGaAs/GaAs quantum wells // Appl. Phys. Lett. 54(17), pp. 1675-1677 (1989).

31. Brierley S. K., Hoke W. E., Lyman P. S., and Hendriks H. T. Photoluminescence characterization of pseudomorphic modulation-doped quantum wells at high carrier sheet densities // Appl. Phys. Lett. 59(25), pp. 3306-3308 (1991).

32. Brierley S. K. Quantitative characterization of modulationdoped strained quantum wells through line-shape analysis of room-temperature photoluminescence spectra // J. Appl. Phys. 74(4), pp. 2760-2767 (1993).

33. Kittel Ch. Quantum theory of solids. - M.: Nauka, 1967.- 492 p. (in Russian)

34. Butov L. V., Kulakovskii V. D., Andersson T. G., and Chen Z. G. Localization effects, energy relaxation, and electron and hole dispersion in selectively doped $n$-type $\mathrm{Al}_{\mathrm{y}} \mathrm{Ga}_{1-\mathrm{y}} \mathrm{As} /$ $\mathrm{In}_{\mathrm{x}} \mathrm{Ga}_{1-\mathrm{x}} \mathrm{As} / \mathrm{GaAs}$ quantum wells // Phys. Rev. B. 42(15), pp. 9472-9479 (1990).

35. Uenoyama T. and Sham L. J. Many-body theory of magneto-optical spectra in doped quantum wells // Phys. Rev. B. 39(15), pp. 11044-11049 (1989).

36. Kulik L. V., Petinova A. V., Kulakovskii V. D., Andersson T. G., Wang S.-M., and Lomsadze A. V. Interaction of aboveFermi-edge magnetoexciton states from different subbands in dense two-dimensional electron magnetoplasma // Phys. Rev. B. 51(24), pp. 17654-17659 (1995).

37. Ando T., Fowler A., Stern F., Electron properties of twodimensional systems. - M.: Mir, 1985.- 416 p. (in Russian)

38. Skolnick M. S., Mowbray D. J., Whittaker D. M., and Smith R. S. Longitudinal-optical phonon and shake-up excitationsin the recombination spectra of semiconductor quantum wells / / Phys. Rev. B. 47(11), pp. 6823-6826 (1993).

39. Nash K. J., Skolnick M. S., Claxton P. A., and Roberts J. S. Phonon sideband of photoluminescence as a probe of exciton states in a quantum well // Phys. Rev.B. 39(8), pp. 5558-5561 (1989).

40. Skolnick M. S., Nash K. J., Tapster P. R., Mowbray D. J., Bass S. J., and Pitt A. D. Free-carrier screening of the interaction between excitons and longitudinal-optical phonons in $\operatorname{In}_{\mathrm{x}} \mathrm{Ga}_{1-\mathrm{x}} \mathrm{As}-\mathrm{InP}$ quantum wells // Phys. Rev. B. 35(11), pp. 5925-5928 (1987).

41. Lyo S. K., Jones E. D., and Klem J. F. Suppression of directtransition phonon side bands in the magnetoluminescence from doped quantum wells // J. Phys.: Condens. Matter. 8(25), pp. L363-L369 (1996).

42. Jaschinski O., Vergöhl M., Schoenes J., Schlachetzki A., and Bönsch P. Observation of Landau levels and excitons at room temperature in $\operatorname{In}_{0.53} \mathrm{Ga}_{0.47} \mathrm{As} / \mathrm{InP}$ quantum wells // Phys. Rev. B. 57(20), pp. 13086-13093 (1998).

43. Kukushkin I. V., von Klitzing K., Ploog K., and Timofeev V. B. Radiative recombination of two-dimensional electrons in acceptor $d$-doped $\mathrm{GaAs}-\mathrm{Al}_{\mathrm{x}} \mathrm{Ga}_{1-\mathrm{x}} \mathrm{As}$ single heterojunctions / / Phys. Rev. B. 40(11), pp. 7788-7792 (1989).

44. Yuan Y. R., Mohammed K., Pudensi M. A. A., and Merz J. L. Effects of carrier confinement in graded $\mathrm{AlGaAs} / \mathrm{GaAs}$ heterojunctions // Appl. Phys. Lett. 45(7), pp. 739-741 (1984). 